\title{
Crowd Sourcing Approach for UAS Communication Resource Demand Forecasting
}

\author{
Chris A Wargo \\ Mosaic ATM., Inc. \\ 540 Fort Evans Road, N.E. Suite \\ 300. \\ Leesburg, VA 20176 \\ 443-994-6137 \\ cwargo@mosaicatm.com
}

\author{
John DiFelici \\ Mosaic ATM., Inc. \\ 540 Fort Evans Road, N.E. Suite \\ 300. \\ Leesburg, VA 20176 \\ 703-582-1772 \\ jdifelici@mosaicatm.com
}

\author{
Aloke Roy \\ Honeywell International \\ 7000 Columbia Gateway \\ Drive, Columbia, MD \\ 21046 \\ (420) 9647336 \\ Aloke.Roy@Honeywell.com
}

\author{
Jason Glaneuski \\ Volpe National Transportation \\ Systems Center \\ 55 Broadway \\ Cambridge, MA 02142 \\ 617-599-3769 \\ Jason.Glaneuski@dot.gov
}

\author{
Robert Kerczewski \\ National Aeronautics and Space \\ Administration \\ NASA Glenn Research Center \\ 21000 Brookpark Road MS 54-2 \\ Cleveland, OH 44135 \\ (216) 433-3434 \\ rkerczewski@nasa.gov
}

\begin{abstract}
Congressional attention to Unmanned Aircraft Systems (UAS) $^{1}$ has caused the Federal Aviation Administration (FAA) to move the National Airspace System (NAS) Integration project forward, but using guidelines, practices and procedures that are yet to be fully integrated with the FAA Aviation Management System. The real drive for change in the NAS will to come from both UAS operators and the government jointly seeing an accurate forecast of UAS usage demand data. This solid forecast information would truly get the attention of planners. This requires not an aggregate demand, but rather a picture of how the demand is spread across small to large UAS, how it is spread across a wide range of missions, how it is expected over time and where, in terms of geospatial locations, will the demand appear. In 2012 the Volpe Center performed a study of the overall future demand for UAS. This was done by aggregate classes of aircraft types. However, the realistic expected demand will appear in clusters of aircraft activities grouped by similar missions on a smaller geographical footprint and then growing from those small cells. In general, there is not a demand forecast that is tightly coupled to the real purpose of the mission requirements (e.g. in terms of real locations and physical structures such as wind mills to inspect, farms to survey, pipelines to patrol, etc.). Being able to present a solid basis for the demand is crucial to getting the attention of investment, government and other fiscal planners. To this end, Mosaic ATM under NASA guidance is developing a crowd sourced, demand forecast engine that can draw forecast details from commercial and government users and vendors. These forecasts will be vetted by a governance panel and then provide for a sharable accurate set of projection data. Our paper describes the project and the technical approach we are using to design and create access for users to the forecast system.
\end{abstract}

\section{TABLE OF CONTENTS}

\section{INTRODUCTION} 1

2. NEED FOR THE FORECASTING TOOL......................2

\footnotetext{
${ }^{1}$ In this paper, UAS and RPA are used interchangeably without intending any difference per se. It is noted that the U.S. DoD and ICAO use RPA while NASA and the FAA use UAS.
}

3. UAXPAN OVERVIEW 2

4. USER FORECAST SERVICES ................................6

7. COMMUNICATIONS DEMAND ...............................9 9

6. OTHER APPLICATION BENEFITS ....................... 12

7. SUMMARY ................................................ 13

ACKNOWLEDGEMENTS......................................... 13

DISCLAIMER....................................................... 13

REFERENCES .......................................................... 14

BIOGRAPHY ............................................................ 14

\section{INTRODUCTION}

The UAS Demand Generator for Discrete Airspace Density (UAXPAN) project allows for a common solution in a complex forecast of the growth and use of numerous unmanned systems and numerous missions types. A key component to solving many of the engineering challenges for integrating UAS into the National Airspace System (NAS) is being able to state within discrete airspace the numbers of forecasted UAS by airframe type and the type of mission or operational use being performed. UAS will exist in a wide range of aircraft and potential uses. The growth in numbers and uses is increasing. The availability of a common cloud based application that can integrate varying growth curves, including the classic sigmoid approach, and linking the geospatial aspects of the UAS flight paths, depending upon commercial needs, will greatly enhance and stabilize major system level analysis problems such as communications spectrum and reuse and safety case analysis. Growth in the use of UAS is a function of locational based business and public use needs. Being able to project the growth in numbers of UAS per airspace of operations requires consensus between different organizations. It also requires the need to have a set of flexible tools able to tune to a changing perspective. 
In similar forecasting, the need for a very detailed configuration is important and time-consuming. Many individuals and organizations need to be able to enter projections in a global version while still allowing individual researchers to set up paradigms for their own, unique forecast.

This paper reports on an innovation in the research and development using a cloud based software system which allows users from all over the globe to create, share, critique, and utilize UA, mission, and growth curve models to produce common data driven forecasts of UA usage patterns and to then have the potential to use plug-in application solvers to further their analysis of the results (e.g. solving for aircraft separation in airspace blocks, sectors, or airspace classes by merging the UAS forecasts with existing historical traffic; or by computing economic projections of capital expenditures based upon UAS.) First the need for the forecasting tool is discussed and the overview of the project system is presented. This is followed by a technical review of the user features and actions being made available. We then present one of the applications tools being developed to use the forecast demand data and translate this data into communications demand. The paper closes with other envisioned applications and a summary.

\section{NEED FOR THE ForECASTING TOOL}

About half of the 2014 report titled "Operational Implications and Proposed Infrastructure Changes for NAS Integration of UAS" analyzed how UASs will interact with the FAA Acquisition Management System (AMS) which is coupled to a supporting capital planning process [1]. The FAA has moved UAS in the NAS Integration project forward, but it is still in the earlier development steps that are the guidelines, practices and procedures of the AMS. The real drive for change in the NAS will come from both operators and government jointly seeking an accurate forecast of UAS demand data. This requires not an aggregate demand, but rather a picture of how the demand is spread across small to large UAS, how it is spread across a wide range of missions and where, in terms of geospatial locations, will the demand appear. In 2012, the Volpe Center performed a study of the overall demand for UASs [5]. However, the underlying demand that was assessed in the study will really appear in clusters of activities that are grouped together by similar missions. These missions take place in a smaller geographical area and then growing outward from those small cells. In general, today there is not a demand forecast that is tightly coupled to the real purpose of the mission requirements (e.g., in terms of the real locations of physical structures; such as windmills to inspect, farms to survey, pipelines to patrol, etc.). Being able to present a solid basis for the demand is crucial in getting the attention of investment, government and other fiscal planners. To this end, Mosaic ATM under NASA guidance is preparing a crowd sourced, demand forecast engine for commercial and government users. This will enable them to drawn upon and be able to share vetted and accurate projection data.

The UAS Demand Generator for Discrete Airspace Density (UAXPAN) project allows for a common solution in a complex forecast of the growth and in the use of numerous unmanned systems and numerous missions types. A key component to solving many of the engineering challenges for integrating UAS into the NAS is being able to state, within discrete airspace, the numbers of forecasted UAS: by airframe type, by the type of mission, or by the operational use being performed. UAS operations will exist in a wide range of potential uses performed by a wide range of aircraft types. The growth in numbers and uses is increasing. As new uses of UASs are being invented today, it is impossible to have a precise demand forecast. The availability of a common cloud based application that can integrate varying growth curves including the classic sigmoid approach as well be able to be constantly updated makes this an important tool to motivate planning and investment. Linking the geospatial aspects of the UAS flight paths depending upon commercial needs will greatly enhance and stabilize major system level analysis problems such as communications spectrum and reuse and safety case analysis. Growth in the use of UASs is a function of locational based business and public use needs. Being able to project the growth in numbers of UASs per airspace of operation requires consensus between different organizations. It also requires the need for having a set of flexible tools able to tune to a changing perspective. In similar forecasting tools, the need for a very detailed input configuration is important and time-consuming. UAXPAN will reduce this complexity while allowing individuals and organizations to be able to enter projections in a global version. Individual researchers can still set up paradigms for their own for unique forecasts.

\section{UAXPAN OVERVIEW}

\section{System Approach}

The NASA purposed innovation in the research and development of a cloud-based software system which allows users from all over the globe to create, share, critique, and utilize UASs, mission, and growth curve models to produce common data driven forecasts of UAS usage patterns will greatly assist UAS adoption. The potential to use plug-in application solvers will further the analysis of forecast results (e.g., control link network design, solving for aircraft separation in airspace, sectors, or airspace classes by the merging the UAS forecasts with existing manned historical traffic; or computing economic projections of capital expenditures based upon UAS regional densities, or job creation from total UAS numbers). The UAXPAN concept is graphically depicted in Figure 1. 


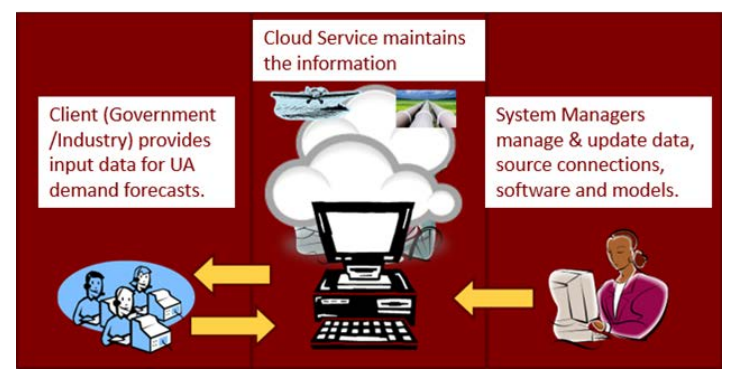

Figure 1. UAXPAN System Overview \& Concept

UAXPAN is scheduled to start Beta Operation in late November 2015. Request for accounts can be sent to: uaxpan_accounts@mosaicatm.com.

\section{Project - Phase I}

Mosaic ATM's Phase I project, UAXPAN, achieved all of its technical objectives and concluded with a successful demonstration of a prototype system at the NASA Glenn Research Center. The significant results stemming from the three objectives set forth in project are as follows:

Objective 1 was the integrated projection over geospatial and time (4D) using practical heuristics for differing UAS growth curves, industry, and missions. Results for this objective include:

- Characterization of UA types into a recommended set of categories ranging from very-small UAS (less than 1 pound) to very large UAS (over 12,000 pounds) and the identification of UA attributes assignable to the UA types for reporting purposes.

- Examination and categorization of industries with the potential for noteworthy UA operational usage and growth, including: agriculture, construction, defense, emergency services, environmental protection, and power generation.

- Assembly of a set of UA missions for identified UQ types, industries, operations, and growth forecasts.

- Investigation into several types of forecast growth curves, including: 'S', 'J', and linear shaped curves.

- Research and approach for airspace projection which combines defined UA missions and forecast curves and projects them geospatially and over time to produce demand forecasts for specified reporting regions. Three specific metric types (max, density, and statistical) were identified, and algorithms for the first two were explored and included in the final prototype

Objective 2 is the development of the initial prototype. For this objective, the following results were obtained:

1. UAXPAN concepts, use cases, and requirements were considered and formulated into a Concept of Operations and System Requirements document. Figure 2 shows the UAXPAN system architecture, some data elements, and user interaction.

2. The full system requirements were scaled down to produce a build plan and sub-requirements for the prototype capable of demonstrating the major concepts and ideas behind the UAXPAN system.

3. The full system requirements were scaled down to produce a build plan and sub-requirements for the prototype capable of demonstrating the major concepts and ideas behind the UAXPAN system.

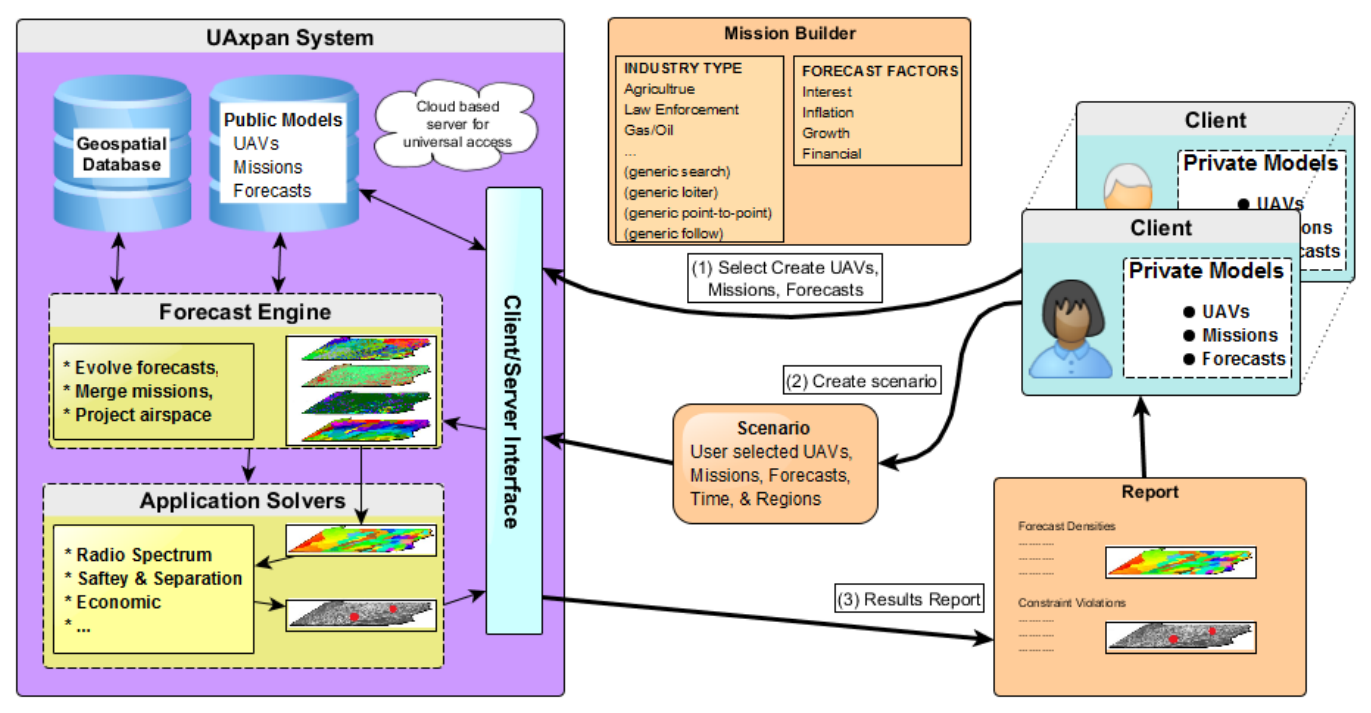

Figure 2. UAXPAN System Architecture and Usage 
4. The full system requirements were scaled down to produce a build plan and sub-requirements for the prototype capable of demonstrating the major concepts and ideas behind the UAXPAN system.

5. Real data derived from government and industry sources was assembled and placed into a PostGIS database for use by the prototype to provide industry, UA mission, and forecast information. Figure 3 shows data examples for windmills, oil pipelines, and Ohio farm usage by county.

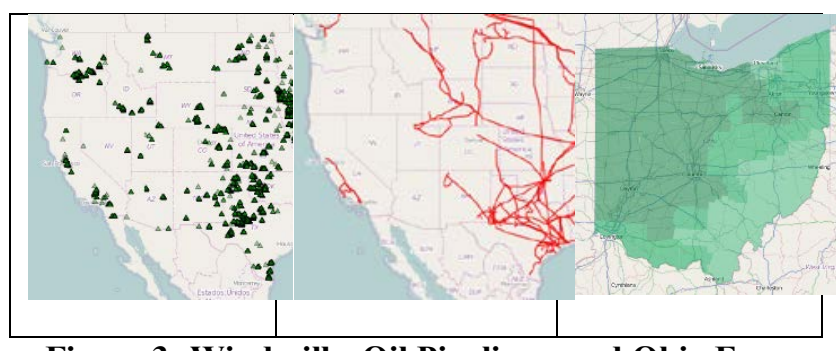

Figure 3: Windmills, Oil Pipelines, and Ohio Farm Usage

6. Development of the prototype was completed and tested on Amazon Web Services for the Cloudbased proof-of-concept. The prototype utilized production ready frameworks allowing for future work to start by extending the existing system, rather than having to start from scratch. Figure 4 shows the results of a scenario demand forecast result over Ohio counties for a combined forecast over several industries and operational missions.

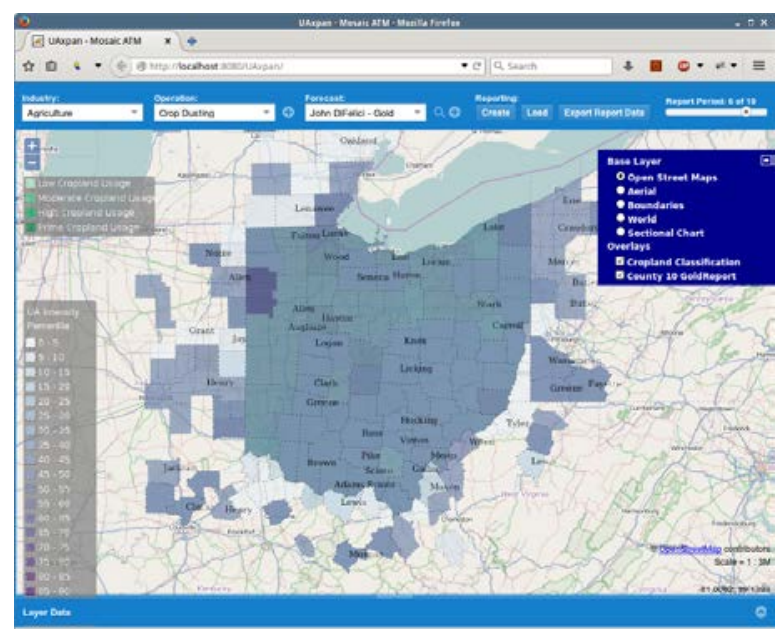

Figure 4: Ohio County Demand Forecast Results

Objective 3 was the analysis of UAXPAN application solvers and assessment of feasibility and use benefits. The results of this objective include an analysis of several different application solvers providing post analysis of demand forecasts and an assessment for the feasibility and benefits from the use of the solvers. The application solvers investigated are:
1. Communication Planning and Support Tool - this application solver ascertains the demand of UAS within geographical areas of the National Airspace System (NAS) to attain bandwidth, control data link congestion and ATC frequency congestion.

2. Air Traffic Control Loading and Planning - this application solver provides TMUs with workload considerations for UAS operations in the NAS.

3. Economic and Environmental Assessment Tool this application solver aids local government officials with UAS demand data and the ability to analyze noise and pollutants created in local regions from UAS operations.

Finally, an analysis of the application solvers investigated was performed, and they were found that they could be both feasible and beneficial.

\section{Project Phase II}

The overall objective of this project is to continue the design, development, and study of the UAXPAN concept. In achieving this objective, we will collaborate with NASA and contribute high-quality work that is relevant and valuable to NASA, advancing NASA's research mission.

In Phase II, our objective is to create a working prototype of the UAXPAN system to establish proof-of-concept and implementation and to also assess performance, marketability, and acceptance. We intend to accomplish this by continuing developing and testing of the Phase I prototype, deploying and running the system in a cloudbased environment, developing pluggable extensions (Application Solvers) to provide additional analysis of the demand forecast results, and finally, by adding application wizards for aiding user creation of missions, operational geometry, and forecasts. We have identified four separate technical objectives for Phase II.

Objective 1 User Feature Tool Completions-The first objective of the Phase II effort is to continue and finish development of the base UAXPAN software system. The Phase I prototype, while functional, was only developed up to the point of being able to demonstrate the basic concepts of a UAXPAN system. The full system still needs to see the development of the following:

- User accounts, data ownership, and system governance.

- New data components: scenarios, data tags, comments and rankings.

- Extensions of existing data components: UA types, industries, operations, and forecasts.

- User input of point and area source operational geometry.

- User defined reporting areas and hexagonal grid support.

- Extend system to accept app solver plugins. 
- Increase flexibility of demand forecast engine: variable time bins and statistics metric.

- Greater flexibility for user selection and entry of operational geometry.

- UI development to support newly created (and extended) features.

- Data tables to handle the newly created (and extended) data elements.

- User guides, documents, and tech support.

Objective 2 Activate Beta Operation- This key objective is necessary to optimize the role of crowd sourcing inputs in a cloud-based hosting approach. It is important to the innovation to be able to assess real user feedback which cannot occur until sufficient users are "online" exercising the system functionality. The Mosaic ATM innovative approach to develop solid forecasts of UAS operations can begin early in the project to test our additional innovation and to add to the research needs that will be received in the feedback from users and the governance group. Accounts will established and approved for users by using outreach and self-identification. Getting into Beta Operation will then also be used to streamline the actions and features needed to support the review and Governance Functions. The governance group will approve the Gold Standard Forecast Scenarios. Three Beta cycles as shown in Figure 5 will be used to accomplish this technical objective. This approach will also allow for users to get results that can support development of standards and policies in the near-term.

Objective 3 Application Solver Implementation-For this objective, three decision support tools, called Application
Solvers, will be created and integrated into the UAXPAN system to provide further analysis of demand forecast data. The Application Solvers to be developed are:

- A communication support and planning tool to facilitate communications loading and spectrum planning for determining radio frequency spectrum to support the control and communications for NAS-wide UAS operations. Mosaic ATM is working with Honeywell, a leading manufacturer and supplier of a broad range of communications, navigation and surveillance radio systems and products to the commercial and military aviation market, whose communications, navigation and surveillance radio products address the needs of virtually all sectors of the civil aircraft market ranging from the larger air transport aircraft to the regional and business aircraft and down to the smaller general aviation aircraft.

- An Air Traffic Control (ATC) loading and planning tool which will support the safe, orderly, and expeditious integration of UAS operations into the NAS via analysis on complexity resulting from said integration and by examining the critical issues of UAS operations on sector staffing, procedures, and airspace capacity.

- An environmental assessment of UAS operations tool capable of extending the demand forecast results with further analysis by performing an environmental impact analysis on noise and atmospheric emissions for UAS operations in the NAS.

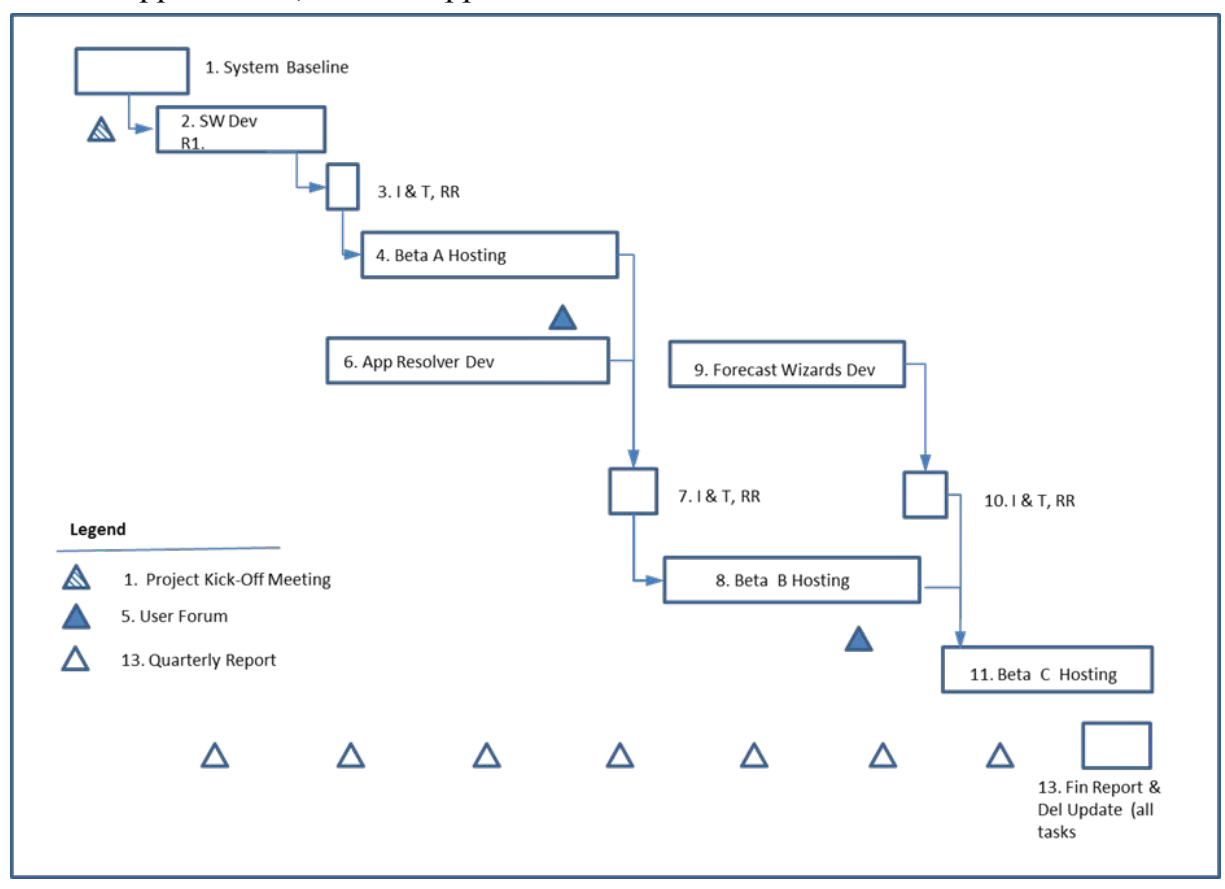

Figure 5. UAXPAN will use an agile development process to capture the innovations of crowd sourcing the UAS Demand Forecast 
Objective 4 User Operation and Forecast Wizard Development-The goal of this objective is to provide the user with an application "Wizard" that guides them through a simple step-by-step process of setting up UAXPAN operations and forecasts. The wizarding software will allow novice users or users without complex mission geometries or forecasts to create operations and forecasts that can be used within the UAXPAN system in the same manner as non-wizard created components. The wizard will:

- Contain simple step-by-step user entry screens.

- Contain in-application tip and help to explain the data entry fields.

- Provide example or default data, where appropriate, to simplify operation/forecast creation.

- Allow for easier definitions of mission geometry in a user-friendly manner.

\section{USER FORECAST SERVICES}

The UAXPAN tool is designed to make it as easy as possible for users to create large, small, local, and/or regional UAS demand forecasts and to then accumulate these partial forecasts into a single merged demand forecast over specified spatial regions and time. Users with specialized knowledge of current or potential future UA operations may utilize the tool by creating one or more of the basic UAXPAN data elements: industry categories, UA types, operations, and operational forecasts. Users interested in constructing local, regional, or national demand forecasts can create scenarios which are populated with existing sets of user created operations and operational forecasts that fit within the needs of the user's scenario. Demand forecast reports can then be created from these scenarios which provide UA demand numbers over user specified regions and over a user specified time period. Figure 6 depicts the basic UAXPAN data elements and how they are utilized.

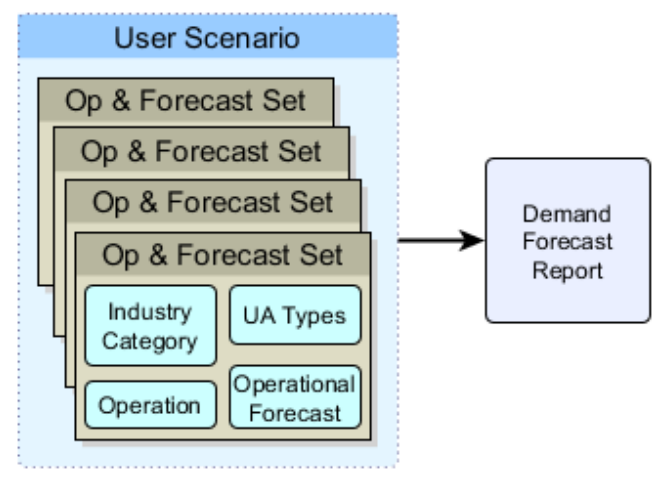

Figure 6. Basic UAXPAN Data Elements
The industry category, UA type, operation, and operational forecast data elements form the basic building blocks of the system. The most basic data element is the industry category element, which is used only as a tag or grouping designator providing a focus for the users and allowing them to categorize their operations and forecasts. UA Types are the second basic data element and initially function as a name tag for demand counts in the forecast reports. While users can define characteristics for the UA types (such as communications bandwidth usage, fuel type, and any other characteristics the user feels are important), for the demand forecasts themselves, only the direct counts by UA type are computed. Nevertheless, starting with the second release of the UAXPAN system and with the development and integration of the Application Solvers, the solvers will be able to make use of the UA characteristic information along with the demand forecasts to provide quantitative information such as: total communication bandwidth usage, areas of congestion, etc.

The operation data element contains information about what, when, and where UA activity occurs. Users associate the operation with an existing industry and then assign one or more UA types to the operation. Together with a descriptive name, these pieces of information define the 'what' or the intent of the operation. For the 'when' portion of an operation, a starting and ending date can be assigned if the activity is to be short lived, or these values can be left unset to reflect an always present activity. Additionally, the frequency property of the operation defines how often the activity occurs and time constraints (if any) can be set to limit the times in which the operations can or cannot occur.

The geometry associated to the activity defines the 'where' of the operation, and UAXPAN tries to make the process of building the operational geometry as reasonable as possible. The geometry for each operation is required in order to produce demand forecasts that are more detailed and specific than those that are accumulated at a national level, or cover large regional areas, or are compiled for an entire industry without any location details. However, defining the geography for an operation can be a time consuming process. It requires searching GIS databases for geographic element definition, gathering and compiling information from disparate sources, and finally merging all that information together and associating it back to the geographic elements.

The UAXPAN system offers several features making the process of defining operational geographic data sets less daunting. Some of these feature include default geographic libraries (states, counties, zip code boundaries, airspace sectors, etc.); the ability to create formulae using fields within data sets; the ability to import custom or user created KML file data files; the ability to join data from disjoint sources by matching on equivalent data feature fields; the ability to, filter data sets and mask geographic areas; and the ability to pull from third party WFS data sources. Additionally, a set of input wizards is being developed to 
add further context, help, and guidance when creating operations and forecasts.

Once a geometry set for the operation has been defined, the workload, the rate of completion, and the UA operational constraints and preferences for the operation can be assigned. The workload for an operation represents the total amount of work to be performed by one or more UAVs. The units of workload can vary by operation type, but some examples include: each individual geometrical element (such as the location of a windmill, radio tower, or other point type sources); the linear distance of geometrical elements (such as pipeline or transmission line data); the area of geometrical elements (such as farmland or county boundaries); or even an absolute number of geometrical elements $(3,5,10)$ contained within the data set. For featured geometric data sets that contain associated data values, the workload units can be combinations of one or more of these values and placed within a user defined formula to create the workload.

The rate of completion for an operation is the time it takes for a single UA to complete a unit of work; thus the total single UA work time required to complete the operation would be the workload divided by the rate of completion. In order to facilitate the assignment of additional UAVs to the operational task, the user can specify the maximum number (Fmax) of UA that can be assigned to perform a unit of work and a flag (Fgroup) to indicate whether or not there is a preference to group multiple UAs on a single task to complete it first, or spread them out over all the work units in the operation as evenly as possible. Figure 7 graphically depicts the operations element, its characteristics associations with the industry, UA types, and geometry elements.

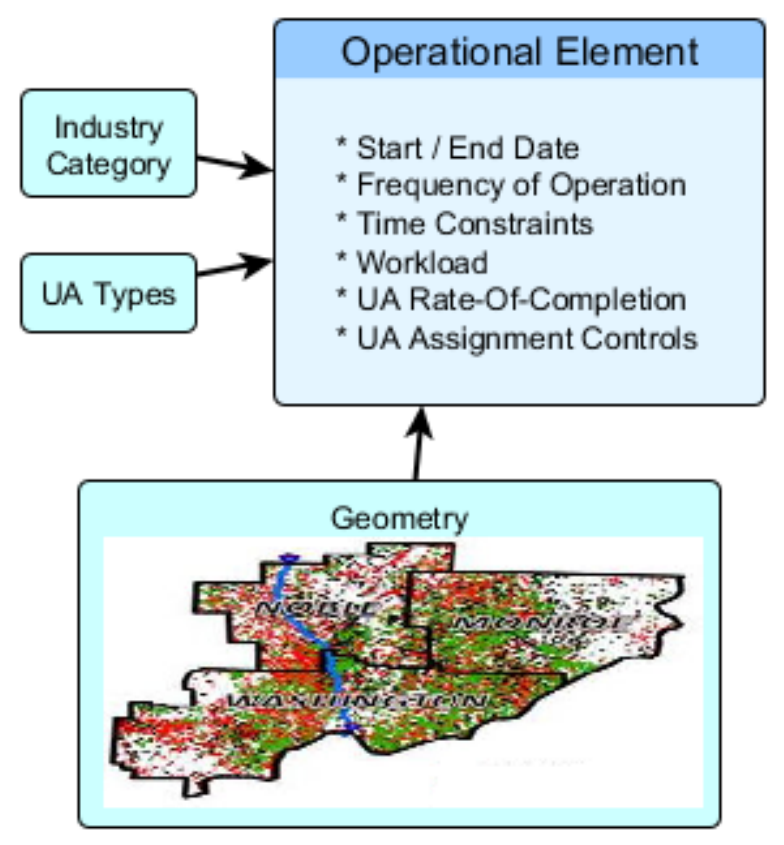

Figure 7. Operation Element Details
The last of the basic data elements is the operational forecast which is used to populate its assigned operation with functional number of UAs over a period of time. The operational forecast is composed of several properties: a start and end date, a forecast curve type, and two or more data points of UA numbers along the curve. The start and end dates define the time period for the forecast, but are clipped by the operation's start and end dates, if defined. If the start and end dates are left blank, then the forecast is valid over whatever range is specified by the operation or by the reporting dates.

The forecast curve type can be one of five curve types: linear, exponential, logistics, Gompertz, or user defined points. The most basic curve is the linear curve, defined by

$$
y(t)=b+k t
$$

This is the standard line formula with a slope of $\mathrm{k}$ and a $\mathrm{y}$ intercept of $\mathrm{k}-\mathrm{y}$ represents the number of UA counts and $\mathrm{t}$ is the time parameter. It requires at least two data points to be defined. If more than two data points are defined, then the line will be determined by a least squares fit to the data.

The exponential curve,

$$
y(t)=b e^{k t}
$$

is a $\mathbf{J}$ shaped curve with an initial value of $\mathrm{b}$ and a growth rate of $\mathrm{k}$. This curve also requires at least two data points to be defined.

The logistics curve,

$$
y(t)=\frac{L}{1+a e^{b t}}
$$

is an $\mathrm{S}$ shaped curve with $\mathrm{L}$ as the upper limit, $\mathrm{a}$ is the location parameter, and $b$ is the shape parameter. This curve requires at least three data points to be defined.

The Gompertz curve is also an $\mathrm{S}$ shaped curve and is another popular curve used in forecasting. The Gompertz curve is defined as

$$
y(t)=L e^{-b e^{-k t}}
$$

where $\mathrm{L}$ is the upper limit to the forecast, b sets the displacement along the $\mathrm{t}$ axis, and $\mathrm{k}$ is the growth rate.

The final curve type is not actually a curve, but rather, a user defined set of UA counts for each time bin. While a curve makes it easy to interpolate UA counts with only two to three points (or guesses) for definition, this user defined option allows the user to specify custom counts at each time 
bin if desired. Figure 8 shows an example of the four main curve types for a 60 month time period.

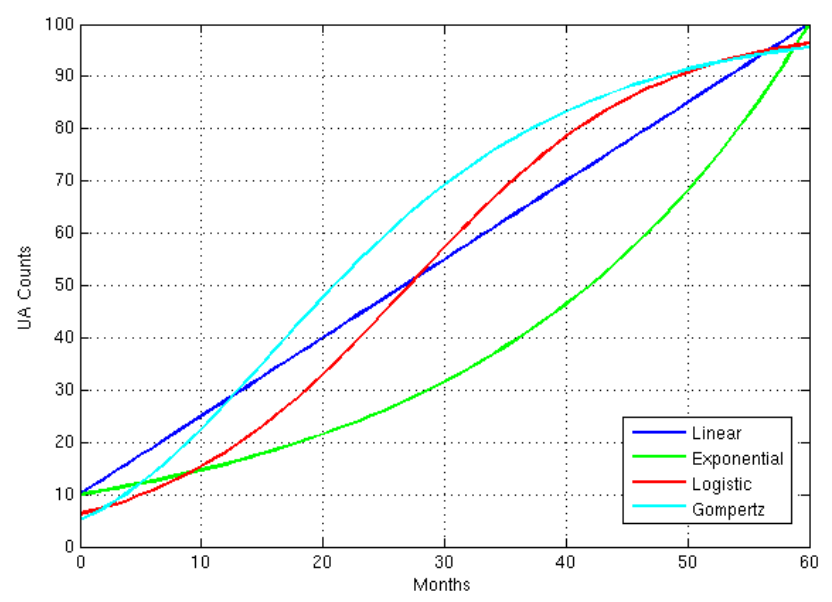

Figure 8. Example Forecast Curves

Users interested in creating demand forecast reports can create a scenario from existing operations and operational forecasts. Comprehensive demand forecasts would include operations / forecasts from all industry categories, while more specialized demand forecasts might include only operations / forecasts from a single industry or operational type. Whatever the user's interests may be, they must first create a scenario and populate it with the operations and operational forecasts of interest to them. After the scenario has been created, the user can then go on to generate a demand forecast report from the scenario.

In addition to a scenario, the demand forecast report requires a start / end date for the reporting period, a bin size parameter which sets both the size and the number of time bins for the reporting period, and an optional reporting area geometry. If the start and/or end dates fall outside an operation's or operational forecast's time bounds (if set), then the affected time bins will receive zero counts for those particular operations. Likewise, reporting areas that fall outside the defined geometry of an operation will receive zero UA counts for those extraneous regions.

Similar to selecting an operation geometry, the reporting geometry can be defined from several possibilities. The user has the option of selecting from one of the predefined geometry boundary sets (US, state, county, zip-codes, airspace sectors), or they can use either a square grid or hexagonal grid with a user defined size. The predefined geometries can be filtered by one or more of their properties (name, size, etc.). Additionally, an outer bounding box can be defined and applied to either a predefined boundary set or to one of the two grid sets.

With all the parameters defined and a scenario selected, the UAXPAN system will iterate through all of the operational and forecast data to project UA counts onto geospatial reporting regions intersecting operational geometries for each time bin in the reporting period. The projection logic keeps track of two metric types for counting purposes: a Max metric and a Density metric. The Max metric keeps track of the maximum possible number of UA counts for each reporting area for each time. It is computed for a given time bin $t$, reporting area, and over every operation as follows:

- Let $\mathrm{Ng}$ be the number of geographic elements from the current operation that intersect with the current reporting area.

- Let $N u$ be the number of UAs assigned to the current operation - determined by the operational forecast curve and its parameters.

- Let Fmax be the maximum number of UAs allowed to work on a single operational work unit / geometry element.

- Let Fgroup (a boolean value) be true if multiple UAs are allowed to operate on a single work unit / geometry element. Otherwise, false if the UA assignments should spread out over all the geographic elements before beginning to double up.

- $\quad$ Let $C$ be the number of computed UA counts for a given reporting area for a given operation - this number will be accumulated over all operations within each time bin $t$ for each reporting area.

If $\mathrm{Nu}$ is less or equal to $\mathrm{Ng}$, then

$$
\mathrm{C}=\mathrm{Nu},
$$

else if Fgroup is false

else

$$
\mathrm{C}=\mathrm{Ng} \text {, }
$$

$$
\mathrm{C}=\min \left(N u, \sum_{k=1}^{N g} F_{\max }(k)\right)
$$

where $k$ runs over all geographic elements in the operation.

The Density metric keeps track of the UA counts for each reporting region by evenly smearing each UA over its operational geometry giving a kind of average of what one may expect to see on any particular day. The Density metric is computed as follows:

- Let $t, N g, N u$, and $C$ be defined as above for the Max metric.

- Let $N G$ be the total number of geographic elements in the operation.

- Let $G L(k)$ be the workload for the kth operational geographic element.

- Let $S g(k)$ be the fraction of the geographic element $k$ that falls within the current reporting region. For point elements, this will always be a value of 1.0 . However, for polyline elements and polygons, this value is determined by the intersecting portion within the reporting region divided by the total 
portion.

If $N u$ is less than or equal to $N g$, then

$$
\mathrm{C}=\mathrm{Nu} * \frac{\sum_{k=1}^{N g(t)} G L(k) * S g(k)}{\sum_{m=1}^{N G} G L(m)},
$$

else

$$
\mathrm{C}=\min (\mathrm{Nu}, \mathrm{Y}(\mathrm{i})) * \frac{\sum_{k=1}^{N g} G L(k) * S g(k)}{\sum_{m=1}^{N G} G L(m)}
$$

where

$$
\mathrm{Y}(\mathrm{i})=\sum_{k=1}^{N g} G L(k) * S g(k) * F_{\max }(k) .
$$

The results of the demand forecast process are depicted above in Figure 4, which show a forecast around the state of Ohio using counties for the reporting regions.

One last feature of importance supported by the UAXPAN system is the governance features. Because UAXPAN is a community supported tool with many potential users, it is important for users to be able to control the visibility of their data, and to be able to provide and receive feedback from other users. Each basic data element, scenarios, and reporting results can be marked by its creator as visible only to the user, one of the user's groups, or open for all of the public to see. Permissions to edit the data elements follows the same pattern as visibility. Data elements visible to a user allow the user to provide feedback in the form of comments and ratings. This feedback can then be utilized by other users when creating scenarios as a filter or subject to review before being included within the scenario.

\section{Communications Demand}

In order to satisfy Objective 3, the application solver for Communications Planning and Support Tool, an existing Honeywell communication system simulation model will be utilized to predict the aggregate bandwidth required to support projected UAS operations. The simulation model will also estimate communication channel loading, one way transit delay by UAS traffic type, and forecast the percentage of lower priority information loss in case adequate spectrum is not available. The simulation model can accommodate multiple UAS classes operating within the same airspace. A graphical user interface permits timephased, dynamic visualization of UAS distribution and channel loading across the National Air Space (NAS) as well as down to a single communication cell.
The scope of the simulation is limited to the following:

- UAS distribution by UAS type across the continental NAS is simulated.

- Candidate communication technologies that have been identified by RTCA Special Committee 228 are modeled for analysis. These technologies include approximations of a C-band $(5 \mathrm{GHz})$ cellular technology for line-of-sight (LOS) operations and a L-band (1 GHz) system for beyond LOS operations.

- The ground network and ground systems are considered as a single cloud entity. Constant delays are accounted in the delay model in the simulation for these entities. Internal network elements are not simulated.

- The data transmission demand estimates in the simulation are limited to macro level traffic generation based on various services expected to be supported by each UAS type. For example, all UAS classes will require communications for command and control while higher end UASs may carry multiple sensors and imaging devices of different criticality levels that will require real-time communications to ground operations center. Data rates for each of these service types are estimated and one or more services is assigned to each UAS class to determine the total data rate demand imposed by that UAS class on the communication system. The simulation does not create actual message transfers across the layers of protocols and end applications.

Figure 10 shows the steps in calculating UAS traffic distribution. The UAS forecasting model requires information about projected UAS operations across the target geographic region. The forecast identifies each UAS by a unique identifier and contains information about its UAS type, mission class, location (latitude, longitude), altitude, airspeed, time of day, origin, destination, and/or operating profile. Based on that input, the model calculates UAS location on a grid-wise map of the NAS. In addition, the model determines UAS distribution on the grid by time.

The overall concept of the simulation model is shown in Figure 9. The tool utilizes the UAS demand forecasts developed using UAXPAN cloud-sourced data as input to the model. This input data drives the UAS mission scenarios across the NAS at various times of day and seasons. These scenarios include all operations related to different kinds of UAS services and for different types of UAS that are forecasted for a given region. As such, mission scenarios provide a basis for estimating the data traffic needed for each UAS type. 


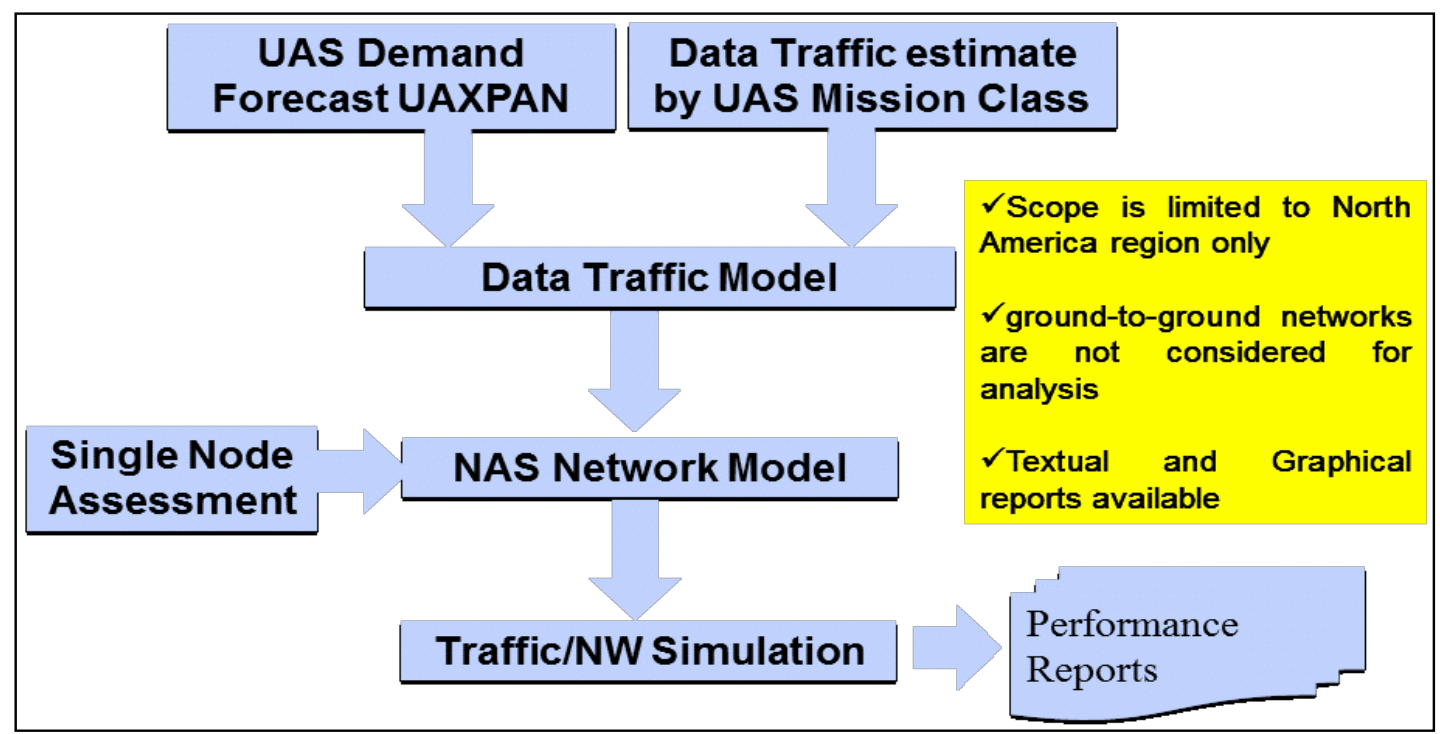

Figure 9. Simulation Model

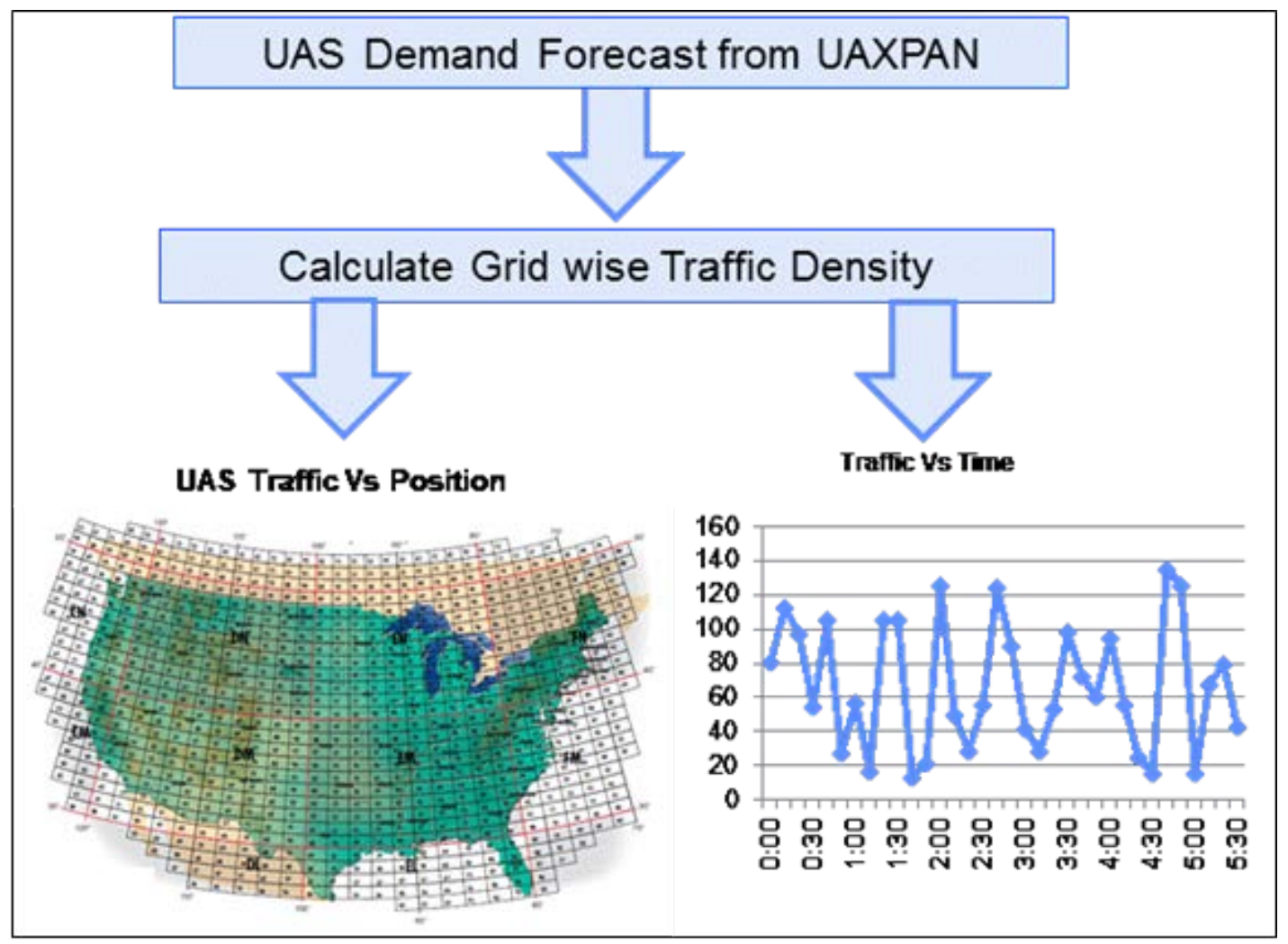

Figure 10. Aircraft Traffic Calculation

The data traffic estimates are categorized into priority levels based on the mission type and supported services. The simulation model supports up to five priority levels whereas each priority is defined to have a set of different Quality of Service (QOS) requirements. Hence the air/ground communication network is considered as an $\mathrm{M} / \mathrm{M} / 1$ system with separate queues for packets with different priority levels, as shown in Figure 11.
In this Priority Queue model, the packets of lower priority start transmission only if no higher priority packet is waiting. The service rate of the channel ' $\mu$ ' is assumed to be the same for different classes. With the arrival rates of different classes given as $\lambda 1, \ldots \lambda \mathrm{k}$, the mean results for latency in the queue, system latency and loss probability are derived. 


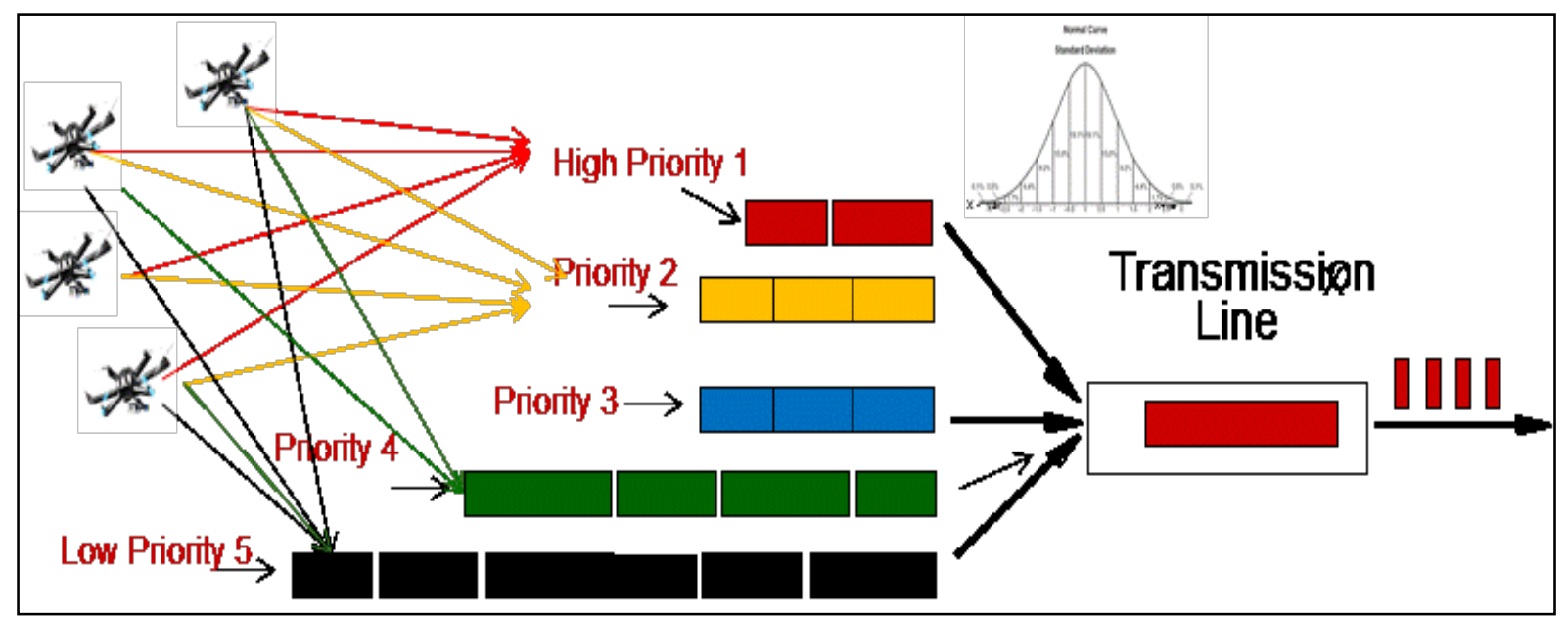

Figure 11. Priority Queuing

The average queuing delay of the kth class is given by following equation:

$$
\begin{aligned}
& \mathrm{Wkq}=\sum \mathrm{kj}=1(\rho \mathrm{j} / \mu \mathrm{j}) /\left(\left((1-\rho 1-\rho 2-\rho 3-\ldots-\rho \mathrm{k})^{*}(1-\rho 1-\right.\right. \\
& \rho 2-\rho 3-\ldots-\rho \mathrm{k}-1))
\end{aligned}
$$

Where, $\rho \mathrm{k}=\lambda \mathrm{k} / \mu \mathrm{k}$; is the fraction of time allocated by server to class $\mathrm{k}$.

The system latency for a given packet is defined as the total time period a tagged packet spends in the system, i.e., the number of time slots between the end of the packet's arrival slot and the end of its departure slot. The average system latency of the kth class is given by the equation:

$$
\mathrm{Sk}=\mathrm{Wkq}+1 / \mu \mathrm{k}
$$

Figure 12 shows how the different classes of packets are prioritized and sent over the channel. The highest priority packets (darker shade, red in color, no diagonal lines, as shown in Figure 12) preempt the lower priority packets ( lighter shade, gold, no diagonal lines, and darker shade, green in color, with diagonal lines as shown in the figure) and are transmitted prior to the lower priority packets. The packets are generated for different classes of UASs based on the estimated rates and sizes. These packets from all the UASs are time stamped and channelized into five First In, First Out (FIFO) buffers with Priority 1 traffic FIFO having highest schedule priority and Priority 5 traffic FIFO having lowest schedule priority. Packets are dropped if the respective FIFO is full. The data traffic scheduler simulates the packet transmissions between the UAS and the tower based on priority queuing scheme. At a given scheduler time instant, the highest priority packets are transmitted first and lower priority packets are scheduled only when there are no higher priority packets to be transmitted. One Transmission Unit (TU) is the smallest data unit at physical layer level that is sent without preemption and scheduler Time Sample (TS) is the time taken to transmit one TU.

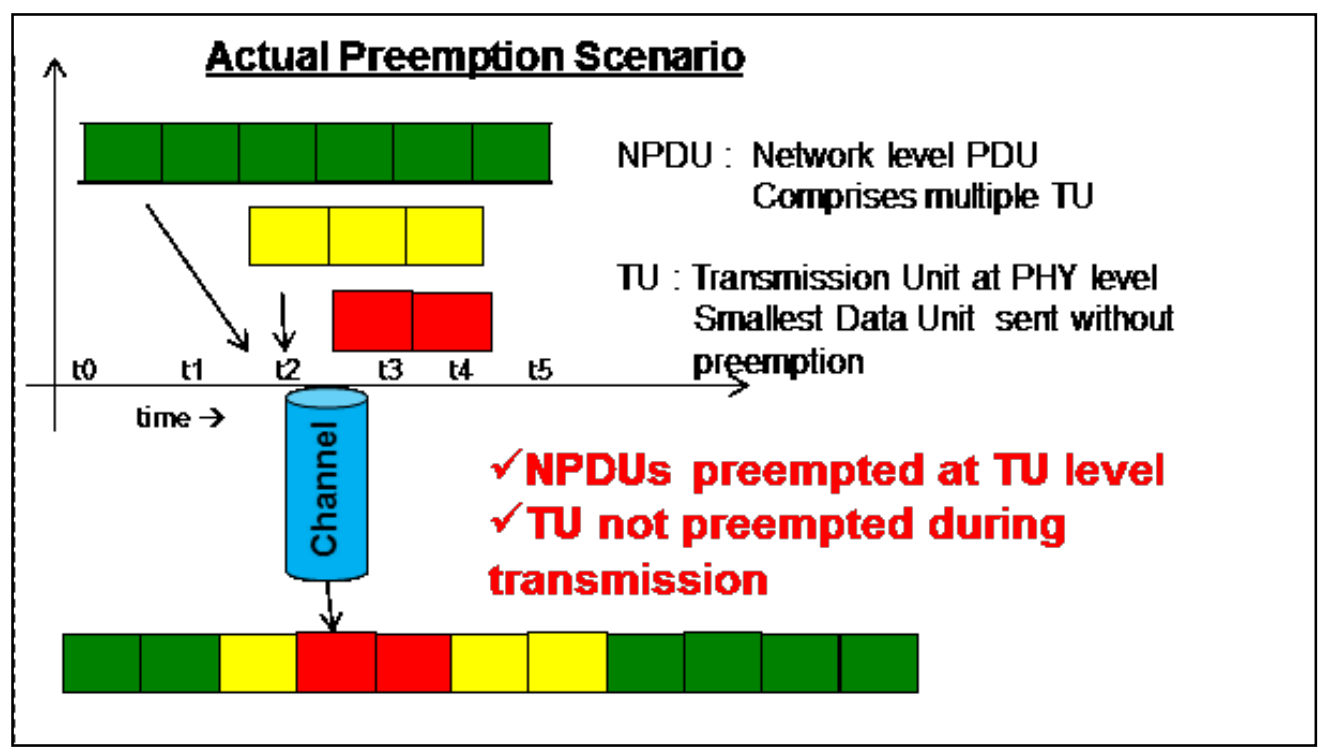

Figure 12. Data Prioritization and Transmission 
The simulation model assumes that the air/ground communication will be cellular in nature, where the service volume of each cell is defined by the physical propagation characteristics of the frequency band and the transmitter power level. In addition, the cellular network architecture assumes that the ground base stations are distributed across the entire NAS region to provide connectivity to the UAS within the service volume of the cell. Each cell is represented by a hexagonal region with a cellular tower located in the center of the hexagonal grid area as shown in Figure 10. A pictorial representation of the cellular service volume is shown in Figure 13.

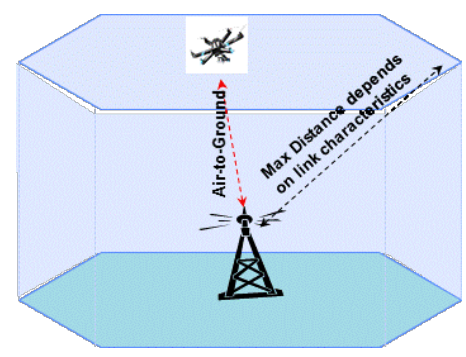

Figure 13. Single Cell Service Volume

The simulator described above acts as the heart of the Communication Planning Tool by generating reports of various network performance parameters viz. peak and average distribution of UASs in each communication service volume, number of packets transmitted, queue latency for each packet, mean latency, percentage of dropped packets by priority level, network utilization for all classes of traffic simulated in the network model, bandwidth utilization for peak concentration of UASs within each communication service volume, etc. These reports can be leveraged by UAXPAN user to develop spectrum deployment and channelization plan. In addition, the UAS distribution by region data can be used for noise, emissions, carbon footprint and other environmental impact assessment.

\section{OTHER APPLICATION BENEFITS}

In order for UAXPAN to successfully incorporate communication assessment and planning support, ATC loading and planning, economic impacts and environmental assessments of UAS operations some assumptions pertaining to the feasibility of this system were made. Table 1 below, summarizes the feasibility and benefits analysis outlined below associated with UAXPAN. While the communication planning and support datalink tool imbedded within UAXPAN ensures datalink coverage will suffice and not be overloaded with demand, it was based upon an RTCA study. Further studies would be necessary to build upon the RTCA work and create a baseline for bit rate and bandwidth usage of various UAS aircraft to enable FAA technical operation to perform the needed analysis. The communication planning and support for ATC ensures controllers' can communicate with UAS to safely, orderly and expeditiously control traffic in the NAS, but there is currently no flight checked altitude to ensure off airway activities that will ensure line of sight with UAS aircraft. While numerous other altitudes are flight checked, they are moot and as a result without a flight checked and certified minimum altitude to maintain there is a risk of losing line of sight and therefore communication with ATC.

The ATC Loading and Planning Decision Support Tool (DST), is designed to ensure ATC sectors will not exceed their MAP values with additional UAS traffic. These MAP values are based upon 15 minute bins of times that aircraft will be entering and exiting the sector, thus with UAS only utilizing abbreviated flight plans that aren't required to be as accurate there is a risk here as well.

While it is a given that UAS operations will result in additional jobs, resulting in more income for communities and a higher quality of life for their citizens, the monetary value is still to be determined. UAXPAN data will add more certainty to economics estimates; since the collected data will show where the operations will transpire and how many operations will be utilized.

The Environmental Impact DST determines how additional noise and emissions could affect the quality of life for citizens located in areas of UAS operations. Unfortunately, while the emissions produced as a result of UAS operations are known, the amount of these emissions and the noise generated are unique to each UAS. As a result, there is a risk associated with this DST as the full extent of the emissions produced and noise generated is still to be determined via the flight time of each UAS and the number of AS flying over each area of operation.

Table 1. UAXPAN Potential Benefits Assessment

\begin{tabular}{|l|l|l|l|}
\hline App Plugin & Feasibility & Assessment & Benefit \\
\hline $\begin{array}{l}\text { Communication } \\
\text { assessment datalink }\end{array}$ & High & $\begin{array}{l}\text { Baseline for bit rate and bandwidth } \\
\text { usage developed and standardized } \\
\text { by RTCA }\end{array}$ & $\begin{array}{l}\text { Ensures datalink coverage will } \\
\text { suffice and not be overloaded } \\
\text { with demand }\end{array}$ \\
\hline
\end{tabular}




\begin{tabular}{|l|l|l|l|}
\hline App Plugin & Feasibility & Assessment & Benefit \\
\hline $\begin{array}{l}\text { Communication } \\
\text { assessment ATC } \\
\text { Comm. }\end{array}$ & Med & $\begin{array}{l}\text { Only certain altitudes are flight } \\
\text { checked and without having } \\
\text { additional altitudes checked there is } \\
\text { a risk }\end{array}$ & $\begin{array}{l}\text { Ensures ATC can communicate } \\
\text { with UAS and safely, orderly } \\
\text { and expeditiously control traffic } \\
\text { in NAS }\end{array}$ \\
\hline $\begin{array}{l}\text { ATC Loading and } \\
\text { Planning }\end{array}$ & Med & $\begin{array}{l}\text { Only abbreviated flight plan being } \\
\text { used, thus less precise location and } \\
\text { time of flight being attained }\end{array}$ & $\begin{array}{l}\text { Ensures ATC will not be } \\
\text { overloaded with UAS to safely, } \\
\text { orderly and expeditiously } \\
\text { control traffic in NAS }\end{array}$ \\
\hline Economic Impacts & Med & Only estimated values at this time & $\begin{array}{l}\text { More income for communities } \\
\text { and better quality of life for } \\
\text { citizens }\end{array}$ \\
\hline Environmental Impact & Med & $\begin{array}{l}\text { Only estimated values for UAS } \\
\text { emissions and noise }\end{array}$ & $\begin{array}{l}\text { Determine how additional noise } \\
\text { and emissions affect quality of } \\
\text { life for citizens }\end{array}$ \\
\hline
\end{tabular}

\section{SUMMARY}

The UAS Integration into the NAS presents a complex challenge to aviation operators, regulators, manufacturers and navigation service providers. Providing solutions that result in proven safe operation of the main segments of the UAS (Control, Unmanned Aircraft, and Support Segment) as integrated into the automation and procedures of the NAS began in earnest in 2004 with the start of civil aviation standards in RTCA SC-203. Meanwhile public agency (military and government agencies) operation has been confined to the strict use of Certificate of Authority airspace which in essence segments the UAS away from other manned aircraft. NASA, the FAA and commercial aviation representatives have struggled to produce a set of working standards even for the targeted UAS subsystems of Command and Control (CC) and, Detect and Avoid (DAA). To provide useable standards that allow manufacturers to seek FAA certification of products, these subsystem must be produced to known technical performance requirements that are set, or constrained, by other subsystems of the UAS, NAS automation platform capacities, shared community use of limited resources (such as protected aviation radio spectrum) or by safety levels acceptable for controller workload capabilities. The engineering solutions imbedded in these products must be based upon a system performance objective that comes from: the realistic forecast of the number of UAs (by type) that are performing defined missions (purpose of flight), UAs in a defined airspace (A through $\mathrm{G}$ ) that is mapped to discrete geospatial locations and being performed during a defined time period. The forecast also needs to cover near-term to far-term horizons. Only once these aircraft per airspace densities are projected, can problems in spectrum reuse and communication channel loading be fully assessed and a detailed design approach be selected. Without this airspace density information, being based upon realistic parameters, a design implementation will be subject to a costly trial and error in the testing of operational acceptability. The cost will be determined in wasted dollars due lack of service or the loss of safety due to pressing of capacity restrictions. For example, NASA in its research to provide prototype $\mathrm{CC}$ avionics needs to baseline its channel sharing and spectrum sharing approaches - using a UA demand forecast that reaches out many years ahead. To do so requires a strong verifiable knowledge of the true demand expected so that avionics performance will be suitable for long periods of service.

Other disciplines involved in the work to define a safe UAS and NAS integration also need accurate forecast data,; such as: safety engineers, airspaces designers, human factor researchers in controller workload, and NAS enterprise architects planning the capacity. By capacity we mean the technical changes needed to systems; such as, ERAM, TAMDR, TFDM, TFM (TBFM), and controller training systems.[2] UAXPAN, as Mosaic ATM proposes, will close the UAS demand knowledge gap and will provide information to allow designers of future UAS subsystems, and of related NAS changes, to be able to offer products that have an extended useful life. The aviation industry needs the UAXPAN results today; as do others that want to forecast business economics leading to determination of job creation or to justify the need for investment dollars.

\section{ACKNOWLEDGEMENTS}

The UAXPAN Project is a Small Business Innovation Research (SBIR) effort that is supported by the NASA Glenn Research Center (Cleveland, Ohio).

\section{DISCLAIMER}

The text and graphics within this paper express only the views and/or opinions of the authors as individuals and do not represent endorsements, commitments or polices of their firm, agency or organization. 


\section{REFERENCES}

[1] John A. Volpe Transportation Systems Center, September 2012, Technical report, Version 0.1, UAS Service Demand: 2015-2035, DOT-VNTSC-DoD-13-01.

[2] John A. Volpe Transportation Systems Center, September 2014, Technical Report, Version 0.1 Research and Analysis Report: Operational Implications and Proposed Infrastructure Changes for NAS Integration of UAS, U.S. Department of transportation, DOT-VNTSC-DOD-14-02.

\section{BIOGRAPHY}

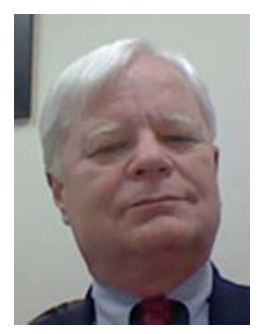

Chris Wargo is a program manager and director business development for Mosaic ATM, Inc. - a firm specializing in air traffic management systems development, unmanned systems and data management systems for the aviation sector. He also leads the Autonomous Systems Group and served as a Chair of the System Engineering Working Group of RTCA SC-203. He has also held positions as President of Computer Network \& Software, Inc., Vice President and General Manager for ARINC, Inc., C3I Program Manager RCA Automated Systems and GE, as well as Systems Engineer for GTE Sylvania, Electronic Systems Group, and the US Army. In his role as a leader in aviation next generation systems engineering, he has participated in numerous ICAO, RTCA, AEEC, IEEE and IATA committees and standards working groups throughout his 30 year defense and aeronautical systems career. He has presented a number of papers and chaired many industry conference sessions related to the CNS and network system programs, project and technologies of the general, business and air transport community. He has a BSEE from the University of Wisconsin and an MS, Systems Engineering, from the University of Southern California, and has attended the Defense Systems Management College and the Advanced Management Program at the Harvard Business School.

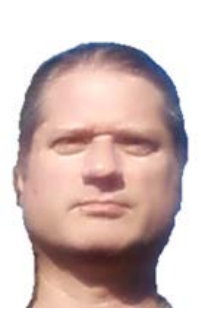

John DiFelici is a principle software engineer for Mosaic ATM, Inc. developing unmanned software systems for the aviation sector. He has worked for over 20 years developing air traffic management, environmental management, and data management systems for the aviation sector. He received a B.S. in Mathematics and Physics from Santa Clara University and his Masters of Physics from the University of Maryland, College Park.

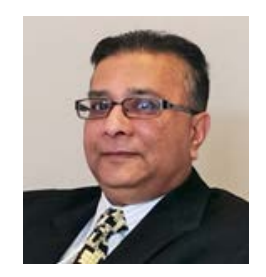

Aloke Roy is a Senior Program Manager with Honeywell Advanced Technology organization. He currently manages data communication, information security and radio technology development programs supporting Honeywell Aerospace. Previously, Mr. Roy was Director of Programs at Flextronics Corporation managing several major telecommunications OEM accounts. In this role, $\mathrm{Mr}$. Roy was responsible for business development, outsourcing, and globalization of hardware design activities supporting large volume contract electronic manufacturing. His prior experiences include various positions at AT\&T Bell Laboratories and ARINC Aviation Systems Division. As Systems Engineering Director at ARINC, Mr. Roy oversaw development of SATCOM, HF, VDL, ATIS, and PDC standards and services. Currently, Mr. Roy chairs ICAO ACP Working Group 'S' and RTCA Special Committee 223, which are developing the Aviation Internet Protocol and Aeronautical Mobile Airport Communication System requirements and operational performance standards. $\mathrm{Mr}$. Roy holds several patents on aeronautical, wireless and secure communications.

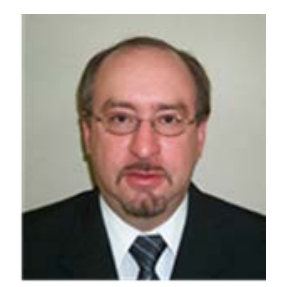

Robert J. Kerczewski has been involved with research and development of satellite and aeronautical communications systems and applications for the Analex Corporation (1982-1986) and NASA (1986-present). He holds a BEE degree from Cleveland State University (1982) and an MSEE degree from Case Western Reserve University (1987). He is currently the Spectrum Element Manager for the NASA's Unmanned Aircraft Systems Integration in the National Airspace System (UAS in the NAS) Communications Sub Project.

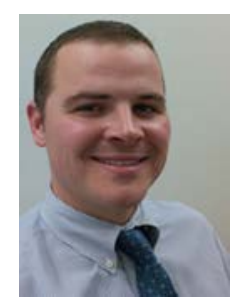

Jason Glaneuski is a Program Manager and Operations Research Analyst in the Air Traffic Management Systems Division at the Volpe National Transportation Systems Center (Volpe Center) in Cambridge, MA. His Division applies information technology and operations research disciplines to enhance the capacity, safety, and security of the National Airspace System. A key component of this work is developing concepts and designing automated decision-support tools and capabilities that provide solutions to existing and anticipated traffic flow issues. Mr. Glaneuski has experience both performing and managing technical work in the areas of traffic flow management (TFM), time-based flow management (TBFM), and unmanned aircraft systems (UAS) sense and avoid (SAA), among others. Mr. Glaneuski is a graduate of Daniel Webster College. 
